\title{
Stress Management Strategies For Students: The Immediate Effects Of Yoga, Humor, And Reading On Stress
}

\author{
Denise Rizzolo, Seton Hall University, USA \\ Genevieve Pinto Zipp, Seton Hall University, USA \\ Doreen Stiskal, Seton Hall University, USA \\ Susan Simpkins, UT Southwestern Medical Center, USA
}

\begin{abstract}
Background: Health science programs can be demanding and difficult for many students, leading to high levels of stress. High levels of stress can have a negative effect on students and subsequently the practicing clinician. Research suggests that yoga, humor, and reading are simple, effective methods to help reduce stress. To date no research compares the acute effects of yoga, humor, and reading in doctor of physical therapy and master's of occupational therapy students. Additionally, it is undetermined if one technique is more effective than the other in reducing stress. Purpose: The purpose of this study was to compare the immediate effects of yoga, humor, and reading on acute stress in students enrolled in doctor of physical therapy and master's of occupational therapy programs. It was hypothesized that following a 30-minute yoga, humor, and reading intervention session, students would demonstrate a reduction of stress on the Daily Stress Inventory (DSI) and a decrease in blood pressure and heart rate. Subjects: Twenty-two students from the School of Graduate Medical Education Doctor of Physical Therapy (DPT) program $(n=14)$ and Master's of Science in Occupational Therapy (OT) program $(n=8)$ volunteered to participate in the study. Methods: At baseline subjects completed a demographic survey. All subjects participated in a yoga, humor, and reading intervention session, once a week on the same day and at the same time for a total of three weeks. Intervention sessions were randomized and counterbalanced across subjects. Before and after each intervention session, stress was measured using the Daily Stress Inventory, systolic blood pressure (SBP), diastolic blood pressure (DSP), and heart rate (HR). Results: A repeated one-way ANOVA indicated that all three interventions significantly decreased $S B P, D B P$, and $H R$ and no one intervention was more effective than the other in reducing these dependent variables $(p=.058, p=.315$ and $p=$ .180 respectively). There was no significant main effect or interaction of the yoga, humor, or reading intervention session on the DSI scores $(p=.362)$. Conclusions: The results of this study indicate that one 30-minute session of yoga, humor, and reading had similar effects in decreasing acute stress in health science students. This finding is important since these interventions resulted in a significant reduction in stress in a relatively short amount of time, allowing educators to begin to consider different stress management strategies to offer to students.
\end{abstract}

Keywords: stress, humor, yoga, reading

\section{INTRODUCTION}

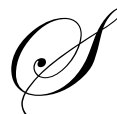

tress is a growing public health concern, affecting many individuals both physically and psychologically (Sobel, 1995). Many individuals are able to cope with small exposures to stress, and some people even become more productive when under pressure (Folkman, 1984; Rowe, 2006). However, it is problematic when stress becomes overwhelming and a person begins to suffer from its adverse consequences including increased anxiety and depression, multiple somatic complaints without an organic cause, or engaging in unhealthy behaviors including smoking, poor dietary habits, and poor sleep habits( Friedman, Sobel, Myers, Caudill, \& Benson, 1995; 
Seyle, 1956; Sobel, 1995). Stress can equally affect all college students; however, the effects of stress are of particular interest in students enrolled in health science programs. Students in health science programs have an intensive academic curriculum and also participate in patient care which can cause an increase in their stress levels (Malathi, \& Damodaran, 1999; Mosley, Perrin, Neral, Dubbert, Grothues, \& Pinto, 1994; O’Meara, Kostas, Markland, \& Previty 1994). While all programs in health science have their own unique stressors, it has been suggested that increased levels of stress are commonly seen in physical therapy students (Frazer \& Echternach, 1995; O'Meara, et al. 1994). For example, Frank and Cassady (2005) found mean stress levels to be higher on the Perceived Stress Scale in 163 doctor of physical therapy (DPT) students compared to a general population of working peers. Not only are levels of stress higher in DPT students, occupational therapy (OT) students experience high levels of stress as well. Tyrell and Smith (1996) found that compared to a group of age matched working adults, occupational therapy students had higher levels of psychological stress on the General Health Questionairre28 (43\% compared to 5\% respectively). PT and OT students consistently report the following factors increase their stress levels: clinical rotations, long hours of studying, lack of free time, and the amount of class work they had to. Furthermore, research suggests that as stress increases so do the number of reported incidences of illnesses, infections, gastrointestinal complaints, neurological, and emotional disorders along with other health problems complete (O'Meara, et al., 1994).

The inability of students to successfully cope with stress may lead to a cascade of negative consequences on both a personal and professional level. Stress can lead to academic decline, poor relationships with peers and family members, and overall dissatisfaction with life (Linn \& Zeppa, 1984). It is postulated that stress begins during the educational process and continues throughout one's professional career (Rowe, 2006). It would therefore be beneficial to encourage the use of stress management techniques at the start of a student's education, thereby assisting them in developing a foundation from which healthy behaviors can be created (Wolf \& Kissling, 1984). While a variety of techniques have been investigated to help reduce stress, three particular adjunctive stress management techniques of interest are yoga, humor, and reading because they are relatively simple and easily accessible. Given that individuals may prefer different stress management techniques or may have different ones available to them assessing if a difference exists in the effectiveness of yoga, humor, and reading is imperative. Furthermore, it would be counter-productive to recommend a technique for stress reduction if the student has no interest in that activity.

Yoga is one of the most recognized forms of exercise, stretching, and mediation. Yoga has been found to modify stress responses and a person's attitude towards stress, while improving self-confidence, increasing one's sense of well-being, and creating a feeling of relaxation and calmness (Malathi, \& Damodaran, 1999; Taylor, 2003). For example, Schell, Allolio and Schonecke (1993) found that after 25 healthy adult females participated in a onehour session of hatha yoga $(\mathrm{n}=12)$ stress scores significantly decreased on the Stress Coping Questionnaire and their heart also decreased compared to a control group ( $n=13)$. Malathi and Damadoran (1999) determined that a onehour yoga program, twice a week, for three months significantly decreased medical students' $(n=25)$ anxiety levels on the Spielberger's Anxiety Scale compared to a control group $(n=25)$. Students who participated in the yoga group also reported improved concentration and an optimistic outlook on life.

While yoga can decrease stress, humor can be a stress modifier as well. The use of humor to help reduce stress has received increased attention by both health care providers and the general public because of its psychological and physiological benefits (Martin, 2001). Humor is defined as the capacity to perceive, appreciate, or express what is funny, amusing, or ludicrous (Humormatters.com Accessed September 5, 2009). To determine the effects of humor on stress, White and Camarena (1989) recruited 93 college students and compared the effects of three different interventions on stress: laughter $(n=26)$, relaxation training $(n=36)$, and a control $(n=33)$, all performed for a period 90 minutes. After the students participated in the interventions for 6 weeks the researchers found that the laughter group demonstrated a decrease in anxiety on the Spielberger State-Trait Anxiety scale, a decrease in systolic blood pressure, and a decrease in heart rate compared to a comparable control group. Similar to these findings, White and Winzelberg (1992) found that after 74 college students participated in 20-minutes of laughter, relaxation training, or a control session, the laughter intervention was as effective as the relaxation session in significantly reducing psychological stress on the Spielberger State Trait Anxiety Inventory and also clinically decreased heart rate. 
While there have been many studies examining the effects of yoga and humor on stress very few have looked at the effects reading has on stress. In one of the few studies on this topic, Jin (1992) found that after a group of 24 healthy adults participated in a stressful task then read for 60-minutes, they experienced a significant reduction in anxiety, heart rate, and blood pressure. Given the limited data on the affects of reading on stress reduction future work investigating its effects are warranted.

Since researchers have determined that some students in health science graduate programs experience high levels of stress which may affect their academic performance it is important for educators to identify a variety of stress management techniques that students can use to manage the stress during graduate school and in the future. Literature on yoga and humor suggests that individually these two methods decrease stress and strengthen coping mechanisms; however, the findings are still limited and many have been equivocal. There has been even less research on the effects of reading on stress. To date no research has compared the acute effects of yoga, humor, and reading on stress in students enrolled in health science programs and more specifically on students enrolled in doctor of physical therapy and master's in occupational therapy programs. Additionally, no research has compared the effects of yoga, humor, and reading on stress to determine if one method is more effective compared to another. Therefore, the purpose of this study was two-fold: (1) to determine the acute effects of yoga, humor, and reading on physical and psychological stress in students enrolled in doctor of physical therapy and master's of occupational therapy programs and (2) to determine if one intervention was more effective then the other in reducing stress. The hypothesis was that immediately following one 30-minute session of yoga, humor, and reading, students would demonstrate a reduction in systolic blood pressure, diastolic blood pressure, heart rate, and psychological stress.

\section{METHODS}

\section{Design}

The study was a quasi-experimental within subject repeated measure design.

\section{Participants}

All students from the Seton Hall University Doctor of Physical Therapy (DPT) and Masters of Science in Occupational Therapy (OT) were asked to participate in this study. The Seton Hall University Institutional Review Board (IRB) approved the research proposal.

Inclusion criteria consisted of any student enrolled in the SHU DPT or OT program. Exclusion criteria included the following: (1) pregnancy, (2) baseline systolic blood pressure less than $90 \mathrm{mmHg}$ and/or diastolic blood pressure less than $60 \mathrm{mmHg}$, (3) a yes response to any question on the Physical Activity Readiness Questionnaire (PAR-Q), (4) students who actively practiced in restorative hatha yoga exercises, similar to the yoga exercises performed in the video instruction, or (5) any subjects with musculoskeletal conditions that would prevent them from practicing light stretching techniques. Based on the above criteria a homogeneous sample of convenience of 22 subjects was recruited.

The dependent variables were psychological stress scores as measured by the Daily Stress Inventory and SBP, DBP, and HR which were measured simultaneously using a Critikon Dinamap XL 9340 Vital Signs Monitor. The independent variables were the 3 interventions: (1) yoga intervention, (2) humor intervention, and (3) reading intervention.

\section{Measurements}

Demographic Data

The demographic survey collected general information from the students and obtained additional sociodemographic data which could account for any confounding effects that were observed on the dependent variables. Questions regarding prescription medication, over the counter medication, herbal supplements, along with whether the student uses a rescue medication during and/or after exercise were included in the survey. 
Hypertensive agents, psychotropic agents and inhaled short-acting bronchodialators may or may not affect psychological stress levels, blood pressure, and heart rate so it was important to determine if the students were using any of these medications (American College of Sports Medicine, 2006).

\section{Stress Survey}

The Daily Stress Inventory (DSI) is a 58 item self-report measure that allows a person to indicate how often a specific stressful event has affected him or her over the past 24 hours (Brantley, Waggoner, Jones, \& Rappaport, 1987). After identifying which events occurred, the individual rates the stressfulness of those events on a Likert scale from 1 ("occurred but was not stressful") to 7 ("caused me to panic"). For the present study only the SUM score was analyzed with the lowest and highest score possible being 0 to 406 respectively. The authors chose only to analyze the SUM score since it demonstrates the total impact the stressors had on the individual in the past 24 hours and subsequently the SUM score can be compared between subjects. The SUM measurement of the DSI is significantly correlated to the Daily Hassles and Uplift Scale $(0.57$ for hassle frequency and 0.56 for hassles intensity) demonstrating concurrent validity. The SUM measurement is correlated to the State-Trait Inventory (0.42) supporting the construct validity of the SUM measurement. The reliability across items for the SUM is 0.87 . Higher scores on the scale indicate a greater psychological stress (Brantley, et al., 1987).

\section{Coping Surveys}

The coping surveys were distributed during the screening session and the results were used as part of another study. The Coping Humor Scale (CHS) is seven item self-reported scale that assesses the degree to which subjects report using humor to cope with stress (Martin, 1996). The Coping Style Scale (CSS) is a 20- item selfreported scale which is part of the Stress Profile published by Western Psychological Services (WPS) (Nowack, 1990; Nowack, 1999). The CSS lists common ways of coping with daily stressors, irritants, annoyances, and challenges.

\section{Physiologic Measurements}

Systolic blood pressure (SBP), diastolic blood pressure (DBP), and heart rate (HR), were taken before and after the interventions simultaneously using an automatic Critikon Dinamap XL 9340 Vital Signs Monitor. SBP, DBP, and HR were measured with the patient in a seated position both feet flat on the floor. Since laughter can initially stimulate the sympathetic nervous system causing an immediate elevation in heart rate and blood pressure, a ten minute delay after each intervention occurred before re-recording SBP, DBP, and HR (Forghieri-Santaella, et al; Fry, 1994).

\section{Interventions}

All subjects participated once in each of the following three interventions over the course of three weeks with each intervention session lasting 30-minutes.

Yoga

Three sections from the Yoga for Meditation DVD, with Rodney Yee, developed by GAIAM, were used. The entire 30-minutes of the DVD is narrated by Rodney Yee and includes soft music in the background. Only clips that practiced restorative yoga, which included light stretching, deep breathing, and meditation were taken. The sections of the DVD that included active aerobic types of yoga techniques were excluded.

\section{Humor}

The subjects selected one of the following videos to watch: The Best of Saturday Night Live with Will Farrell, Chris Farley, or Jimmy Fallon. 


\section{Reading}

During the reading intervention session the subjects read articles about historical events and innovative technology. Articles were taken from Newsweek and various internet sites. The material was non-provocative and it did not relate to any health maintenance topics.

\section{Procedure}

During a screening visit students completed the PAR-Q followed by blood pressure measurements to determine inclusion status. If the student met the inclusion and exclusion criteria, they then completed the baseline scales including the demographic survey, the Coping Style Scale, and Coping Humor Scale. Following the scales the students were assigned to a different intervention session sequence to control for order effects.

During the three intervention sessions stress was initially assessed using the Daily Stress Inventory, SBP, DSP, and HR. Immediately following the intervention the DSI was completed and 10 minutes later SBP, DSP, and HR were recorded. Each subject was exposed to each of the independent variables and performance was compared across treatment conditions within each subject to help control for intersubject differences. Over a three week period each student participated each of three interventions. All data and intervention sessions were recorded and performed on the same day of the week and at the same time of day in order to control for possible weekly and daily fluctuations in SBP, DSP, HR and stress levels. Data collection occurred during the first four weeks of the semester in attempt to avoid the confounding effects of midterms

\section{Data Analysis}

Frequency counts and descriptive statistics were used to analyze the demographic data. After mean psychological stress scores were calculated from the DSI they were found to have a high standard deviation suggestion there scores were extremely variable. Therefore the scores from the DSI were entered into a logarithmic regression model in order to normalize the data prior to the ANOVA analysis. To determine the effect of yoga, humor, and reading on stress a One-Way Within Subjects Repeated Measures ANOVA was computed using a p value of less then 0.05 to determine significance (Munro, 2005; Portney \& Watkins, 1993). The Statistical Package for the Social Sciences (SPSS) software, version 15.0 for Windows (2007), was utilized for all computations.

\section{RESULTS}

\section{Demographic Profile}

Twenty-four full-time students with a mean age of 23 years (range 20 to 37 years) volunteered for the study. After the initial screening visit, one student withdrew at the beginning of the first session for an unspecified reason. One student did not complete the yoga intervention nor yoga post-tests due to cervical pain unrelated to the present study. Eight students (36\%) used prescription medications including birth control, cetirizine $\mathrm{HCl}$ (Zyrtec), and one student took sertraline hydrochloride (Zoloft). Two students (9\%) used over the counter medication, none of the students used medication before or after exercise, and $2(9 \%)$ students stated they used some form of herbal supplements. Seventeen (77\%) students exercised at least three days a week, for an average of 50 minutes per session. Types of exercise included predominately running, walking, elliptical machine, and strength training. Only 3 students (14\%) participated in an aerobic form of yoga once per week. The 12 students (54\%) who reported drinking alcohol on average consumed only one drink per week. The results indicated that the sample consisted of healthy college students that exercise regularly, do not abuse alcohol nor smoke tobacco, and do not use medications to control blood pressure. A summary of the remaining demographic and personal habits is presented in Table 1 . 
Table 1: Demographic Data

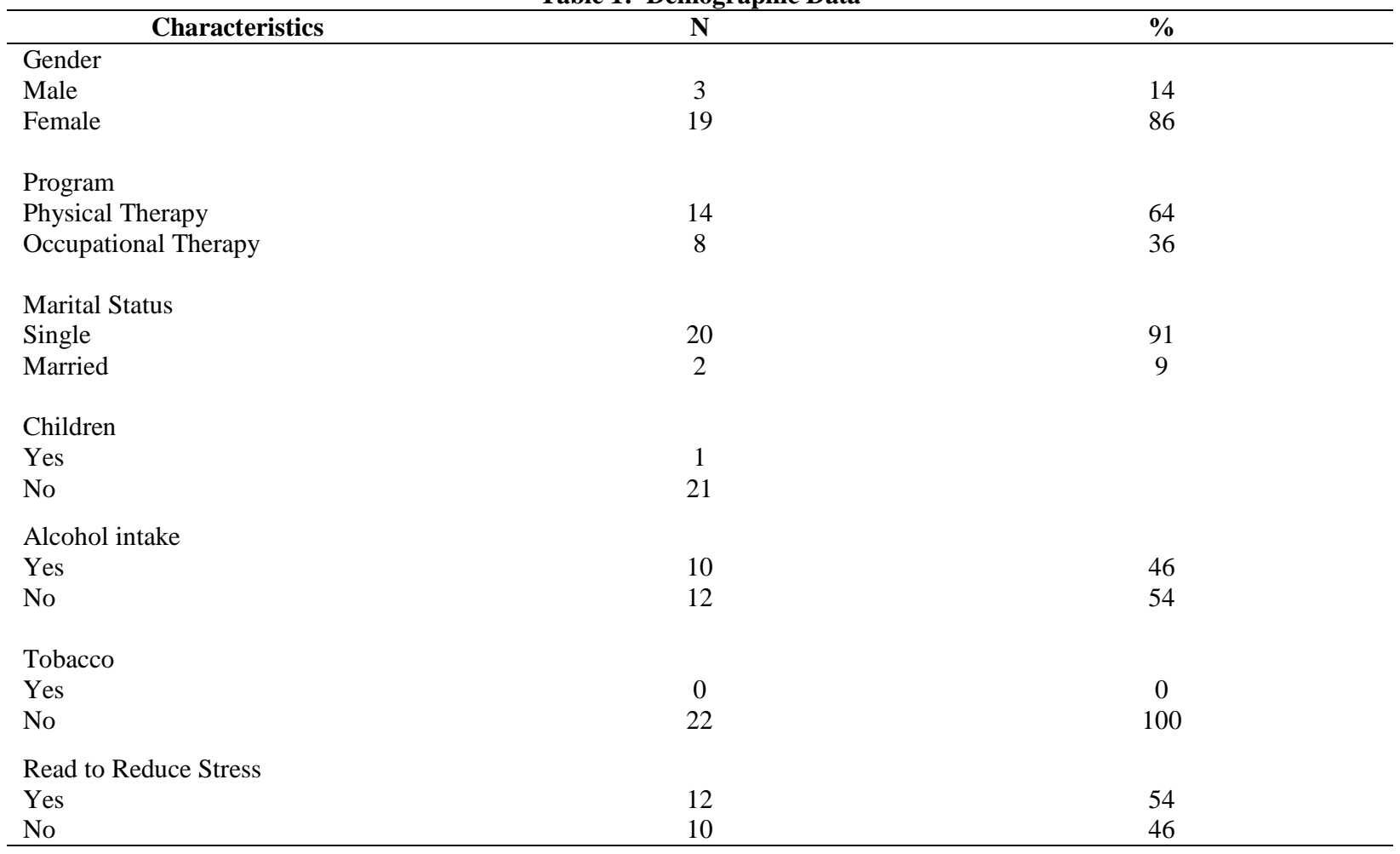

\section{Psychological and Physiological Measures}

Calculated means and standard deviations for the DSI scores SBP, DBP, and HR appear in Table 2. The mean blood pressure and heart rate levels indicate that these variables were normal to low at baseline, suggesting that this sample consisted of healthy young students (Chobanian, et al. 2003).

Table 2: Summary of Pre and Post Mean DSI Scores, Blood Pressure and Heart Rate by Intervention

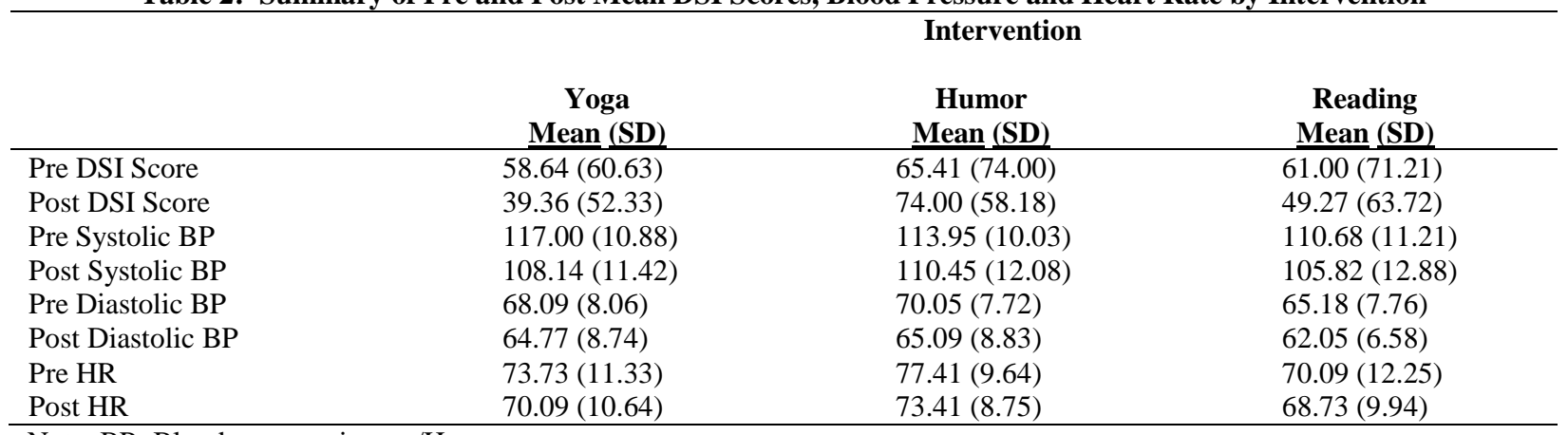

Note. $\mathrm{BP}=\mathrm{Blood}$ pressure in $\mathrm{mm} / \mathrm{Hg}$

$\mathrm{HR}=$ Heart rate in beats/min

A repeated measures analysis of variances (ANOVA) indicated there was no significant main effect of intervention on the DSI scores. A significant main effect of time on DSI scores was found $(\underline{F}(1)=18.56, \underline{p}=.000)$. However, there was no significant interaction between intervention $\mathrm{x}$ time, indicating that no one intervention reduced DSI scores more than another. 
There was a significant main effect of the intervention $(\underline{F}(2)=5.27, \underline{p}=.009)$ and time $(\underline{F}(1)=32.12, \underline{p}<$ $.05)$ on SBP. The interaction between intervention $\mathrm{x}$ time approached but did not reach significance $(\underline{\mathrm{F}}(1.511)=$ $3.403, \mathrm{p}=.056$ ), indicating that no one intervention reduced SBP more than another.

There was a significant main effect of the intervention $(\underline{F}(2)=3.48, \underline{p}=.04)$ and time $(\underline{F}(1)=41.90, p=$ $.000)$ on DBP. Yet, there was no significant interaction between intervention $x$ time, indicating that no one intervention reduced DBP more than another.

A significant main effect of the intervention $(\underline{F}(2)=5.56, \underline{p}=.008)$ and time $(\underline{F}(1)=12.33, \underline{p}=.002)$ on $\mathrm{HR}$ was also found. Yet, there was no significant interaction between intervention $\mathrm{x}$ time, indicating that no one intervention reduced HR more than another.

\section{DISCUSSION}

The results suggest that one 30-minute session of yoga, humor, and reading acutely reduced physiological and psychological stress in students enrolled in graduate DPT and OT programs. Specifically, participation in a single session of yoga significantly reduced students' SBP, DSP, and HR. The observed change in the DSI scores and the significant decrease in the physiologic variables noted after the yoga session could be attributed to the decrease in sympathetic arousal that occurs when subjects participate in this type of activity (Schell \& Allolio \& Schonecke, 1993). In the present study the light stretching and quiet mediation during the yoga intervention session could have caused a decrease in physiologic parameters, which in turn may have contributed to a decrease in the psychological perception of stress.

Similar to the decrease in stress seen after the yoga intervention session, the humor intervention session clinically decreased psychological and physiologic stress. The results are consistent with some of the findings White and Winzelberg (1992) who found that after a group of college students watched a humorous video for 20 minutes a significant decrease on the State-Trait Anxiety Inventory (STAI) occurred along with an observed but not significant reduction in blood pressure. The authors (1992) believed blood pressure did not significantly decrease because during the laughter session, the subjects laughed for less then four minutes, which may have been an insufficient amount of time to cause a decrease in sympathetic arousal. In comparison, in the present study, the primary researcher observed that almost all the students laughed throughout the entire length of the 30-minute video which could explain the significant decrease in SBP, DBP, and HR observed.

After the reading intervention session the students also had a decrease in the stress variables. These results were not unexpected since over half the students admitted using reading to help reduce stress. However, even though 12 of the 22 students read to reduce stress, this did not significantly influence the effect of any intervention on the dependent variables $(\mathrm{p}=0.66)$. The students may have found the neutral reading material to be relaxing, thereby decreasing the sympathetic nervous system arousal and resulting in a reduction of stress. Since there are limited studies on the effects of reading on stress, more research is needed to clearly determine why reading reduces stress levels.

Findings from the present study on graduate health science students demonstrate that individually one 30minute yoga, humor, and reading session are equally effective in acutely reducing stress. Thus, supporting the notion that there are multiple methods one can utilize to help reduce the negative effects of a stressful situation. Since time constraints are one of the most frequently cited reasons for high stress levels reported by health science students, 30minutes of one of these techniques can be easily incorporated into their schedule without diverting a large amount of time from their studies. Finally, the combination of a significant reduction in acute stress in a short amount of time is of importance because yoga, humor, and reading can be suggested to students for immediate stress reduction before examinations, practicals, presentations and even during a highly stressful day.

\section{Limitations and Future Research}

The author recognizes there are limitations to the present study. First, a sample of convenience was recruited that included DPT and OT students from one university, thus limiting the generalizability of the results. 
The sample size was smaller than initially projected do to the limited recruitment period which was designed to attempt to avoid the confounding effects of midterms. Although the study was done during the first four weeks of class to account for increased stress levels as the semester progresses, it was impossible to control for outside influences that may have increased or decreased the students' stress levels. Additionally, an unequal number of students from these two programs volunteered for the study, however, their stress levels were found to be similar at baseline. In the future, physiological measurements should be taken throughout the intervention and after the completion of the intervention to determine short and long-term effects of yoga, humor, and reading on stress.

\section{CONCLUSION}

Programs in health science place students in demanding, fast-paced, and stressful academic environments. It is well known that students in health science programs perceive their academic environment to be stressful (Frazer \& Echternach, 1995; O'Meara et al., 1994). While professors teach students pathology, diagnosis, and treatment they do not generally teach them how to cope with the stress frequently encountered in many health science professions. The stress they face during their education may continue throughout their careers, ultimately leading to burnout (Balogun et al., 2002). Given the high prevalence of burnout among physical therapists and occupational therapists as a whole, it is important for programs to begin to focus their attention on the students stress so they can learn how to cope and manage stress before they enter their professional fields (Rowe, 2006). In addition to preparing students for their work as clinicians, educators should help student identify stressors. Early self identification of stress is important in order to develop coping methods essential for managing the high stress position of a professional practitioner. Hopefully by dealing with stress effectively as a student and then as a practicing clinician, some of the adverse physiological and psychological effects of stress will be mitigated.

\section{AUTHOR INFORMATION}

Dr. Rizzolo is an Assistant Professor at the Seton Hall Physician Assistant program. She received her PhD from the Graduate Programs in Health Sciences at Seton Hall University in 2008. She currently works as a Physician Assistant at the Care Station in Springfield, New Jersey. Her research interests focus on stress management and adjunctive techniques to management specifically, yoga and humor.

Dr. Pinto Zipp is Associate Professor and Chair of the Department of Graduate Programs in Health Sciences at Seton Hall University. She received her EdD from Teachers College Columbia University in 1996. She currently teaches management of neuromuscular problems in the Doctor of Physical Therapy program. Her research interests focus on, a) effects of performing dual tasks on walking performance and postural sway in children and adults, and b) curriculum design issues including the use of mind mapping and video based cases in professional education for the promotion of clinical decision making skills.

Dr. Simpkins is Associate Professor in the Department of Physical Therapy,UT Southwestern Medical Center. She received her EdD from Teachers College Columbia University in 2000. She currently teaches management of pediatrics neuromuscular problems in the Physical Therapy program. Her research interests focus on, a) effects of obstacles on walking performance in children, and b) curriculum design issues.

Dr. Stiskal-Galisewski is an Associate Professor and Chair of the Seton Hall University Physical Therapy Program. She received her PhD from the Graduate Programs in Health Sciences at Seton Hall University in 2003. Her research interests are in osteoarthritis, rheumatoid arthritis, and the effects on Tai Chi on these conditions.

\section{REFERENCES}

1. American College of Sports Medicine. (2006). Resource Manual for Guidelines for Exercise Testing and Prescription ( $5^{\text {th }}$ ed). Baltimore, MD: Lippincott Williams \& Wilkins.

2. Brantley, P. J., Waggoner, C. D., Jones, G. N., \& Rappaport, N. B. (1987). A daily stress inventory: development, reliability, and validity. Journal of Behavioral Medicine, 10(1), 61-74.

3. Balogun, J.A., Titiloye, V., Balogun, A., Oyeyemi, A., \& Katz, J. (2002). Prevalence and determinants of burnout among physical and occupational therapists. Journal of Allied Health, 31(3), 131-139. 
4. Chobanian, A. V., Bakris, G. L., Black, H. R., Cushman, W. C., Green, L. A., Izzo, J. L. et al., (2003) Seventh report of the joint national committee on prevention, detection, evaluation, and treatment of high blood pressure. Hypertension, 42, 1206-1252.

5. Folkman, S. (1984). Personal control and stress and coping processes: a theoretical analysis. Journal of Personality and Social Psychology, 46, 839-852.

6. Forghieri-Santaella, D., Aparecida-Araujo, E., Coelho-Ortega, K., Tinucci, T., Mion, D., Negrao, C.E., \& Lucia de Moraes-Forjaz, C. (2006). After effects of exercise and relaxation on blood pressure. Clinical Journal of Sports Medicine, 19(4), 341-347.

7. Frank, L.M. \& Cassady, S.L. (2005). Health and wellness in entry-level physical therapy students: are measures of stress, anxiety, and academic perfromance related? Cardiopulmonary Physical Therapy Journal, 16, 5-16.

8. Frazer, G. H, \& Echternach, J. L. (1991). Response of physical therapy students to stress indicators. Journal of Physical Therapy Education, 5, 72-77.

9. Friedman, R., Sobel, D., Myers, P., Caudill, M., \& Benson, H. (1995). Behavioral medicine, clinical health psychology, and cost offset. Health Psychology, 14(6), 509-518.

10. Fry, W. F. (1994). The biology of humor. Humor, 7(2), 111-126. Humormatters.com (2009). Definitions of humor. Retrieved September 7, 2009 from http://humormatters.com.

11. Jin, P. Efficacy of tai chi, brisk walking, meditation, and reading in reducing mental and emotional stress. Journal of Psychosomatic Research, 36(4), 361-370.

12. Linn, B. S., \& Zeppa, R. (1984). Stress in junior medical students: Relationship to personality and performance. Journal of Medical Education, 59(1), 7-12.

13. Malathi, A., \& Damodaran, A. (1999). Stress due to exams in medical students-role of yoga. Indian Journal of Physiology and Pharmacology, 43(2), 218-24.

14. Martin, R. A. (1996). The situational humor response questionnaire and coping humor scale: a decade of research findings. Humor, 9, 251-272.

15. Martin, R. A. (2001). Humor, laughter and physical health: methodological issues and research findings. Psychological Bulletin, 127(4), 504-519.

16. Mosley, T. H., Perrin, S. G., Neral, S. M., Dubbert, P. M., Grothues, C. A., \& Pinto, B. M. (1994). Stress, coping, and well-being among third year medical students. Academic Medicine, 69(9), 765-767.

17. Munro, B. H. (2005). Statistical Methods for health care research. $5^{\text {th }}$ ed. Philadelphia, PA, Lippincott Williams \& Williams.

18. Newman, M. G., \& Stone, A. A. (1996). Does humor moderate the effects of experimentally-induced stress? The Society of Behavioral Medicine. 18(2), 101-109.

19. Nowack, K. M. (1990). Initial development of an inventory to assess stress and health risk. American Journal of Health Promotion, 4, 173-180.

20. Nowack, K. M. (1999) Stress Profile Manual. Western Psychological Services, Los Angeles, CA.

21. O’Meara, S., Kostas, T., Markland, F., and Previty, J. C. (1994). Perceived academic stress in physical therapy students. Journal of Physical Therapy Education, 8, 71-74.

22. Portney, L.G., \& Watkins, M.P. (1993). Foundations of clinical research. $1^{\text {st }}$ ed. Stanford, CT: Appleton \& Lange.

23. Rowe, M. M. (2006). Four-year longitudinal study of behavioral changes in coping with stress. American Journal of Health Behavior, 30(6), 602-612.

24. Schell, E. J., Allolio, B., \& Schonecke, W. (1993) Physiological and psychological effects of hatha-yoga exercise in healthy women.International Journal of Psychosomatics, 41, 46-52.

25. Selye, H. (1956). Stress of Life. New York, NY: McGraw-Hill Bock Company.

26. Sobel, D. S. (1995). Rethinking medicine: improving health outcomes with cost-effective psychosocial interventions. Psychosomatic Medicine, 57, 234-244.

27. Statistical Package for the Social Sciences, 15.0 for Windows (2007). Chicago Illinois: SPSS, Inc.

28. Taylor, M. J. (2003). Yoga Therapeutics: an ancient, dynamic systems theory. Techniques in Orthopedics, 18(1), 115-125.

29. Tyrrell, J. \& Smith, H. (1996). Levels of psychological distress among occupational therapy students. British Journal of Occupational Therapy, 59(8), 365-371.

30. White, S. \& Camarena P. (1989). Laughter as a stress reducer in small groups. Humor, 2(1), 73-79.

31. White, S. \& Winzelberg, A. (1992). Laughter and stress. Humor, 5(4), 343-355. 
32. Wolf, T. M., \& Kissling, G. E. (1984). Changes in life style characteristics, health, and mood of freshman medical students. The Journal of Medical Education, 58, 806-814.

\section{NOTES}

